\title{
Comparison of averages of flows and maps
}

\author{
Z. Kaufmann ${ }^{1, *}$ and H. Lustfeld ${ }^{2, \dagger}$ \\ ${ }^{1}$ Department of Physics of Complex Systems, Eötvös University, P.O. Box 32, H-1518 Budapest, Hungary \\ ${ }^{2}$ Institut für Festkörperforschung, Forschungszentrum Jülich, D52425 Jülich, Germany
}

(Received 9 March 2001; published 26 October 2001)

\begin{abstract}
It is shown that in transient chaos there is no direct relation between averages in a continuous time dynamical system (flow) and averages using the analogous discrete system defined by the corresponding Poincaré map. In contrast to permanent chaos, results obtained from the Poincaré map can even be qualitatively incorrect. The reason is that the return time between intersections on the Poincare surface becomes relevant. However, after introducing a true-time Poincaré map, quantities known from the usual Poincaré map, such as conditionally invariant measure and natural measure, can be generalized to this case. Escape rates and averages, e.g., Liapunov exponents and drifts, can be determined correctly using these measures. Significant differences become evident when we compare with results obtained from the usual Poincare map.
\end{abstract}

DOI: 10.1103/PhysRevE.64.055206

Extensive investigations of chaotic systems in recent years have demonstrated the great importance of transient chaos, due mainly to its connection with transport phenomena [1-3] and chaotic advection [4], possibly associated with chemical reactions [5]. In most chaotic systems for certain purposes, it is sufficient to know the intersection points of the trajectories with a chosen surface $P$, the so-called Poincaré surface. In the case of $N$-dimensional phase space, $P$ is $N-1$ dimensional. Using a coordinate system on $P$, and finding the connection between the successive intersections $\mathbf{x}_{n}$ and $\mathbf{x}_{n+1}$, the Poincare map (PM) can be constructed as

$$
\mathbf{x}_{n+1}=\mathbf{f}\left(\mathbf{x}_{n}\right) \text {. }
$$

The behavior of the system can then be studied by iteration of this map. The advantages of the use of PM are (i) it is discrete; (ii) it has smaller dimension. Its disadvantage is the absence of the close connection between the number of intersections $n$ and the time $t$, since the return time $\tau$ between two intersections depends generically on where a trajectory intersects. One can keep this information by completing the $\mathrm{PM}$ with the equation

$$
t_{n+1}=t_{n}+\tau\left(\mathbf{x}_{n}\right) .
$$

We call this extended map the true-time Poincare map (TPM).

Usually, one reduces to the PM by the following argument: The total time after $n$ iterations is given by the sum of the corresponding return times $\tau(\mathbf{x})$. It is generally assumed that for large $n$ and for typical trajectories, the terms in the sum can be replaced by their average over the invariant density $\rho_{P}$ of the map. The sum then becomes a product [6]:

$$
t=n\langle\tau\rangle,\langle\tau\rangle=\int_{P} d x \rho_{P}(\mathbf{x}) \tau(\mathbf{x})
$$

\footnotetext{
*Electronic address: kaufmann@complex.elte.hu

†Electronic address: h.lustfeld@fz-juelich.de
}

PACS number(s): 05.45.-a, 05.70.Fh
Based on this connection, averages of the map (using $n$ for time) and the flow (using the real time $t$ ) would be simply related by a time scale. This is explicitly shown for general averages in case of permanent (nontransient) chaos [7].

We demonstrate in this paper that, in contrast to permanent chaos, the situation is quite different for transient chaos. Not only should $\langle\tau\rangle$ in Eq. (3) be changed, but averages of the map and the flow (or of the TPM representing it) are not anymore related by a time scale. The situation is somewhat reminiscent of the case when, instead of simple averages, the decay rates of correlations are considered. Even in permanent chaos, these show a discrepancy in nonideal situations [8]. To proceed in a correct manner, we must start with the TPM, which contains all the information needed for the long-time behavior of the system and from which we can derive all necessary formulas. Finally, we compare these with the corresponding ones of the PM by setting $\tau(\mathbf{x})$ $=\langle\tau\rangle$. The use of the PM is sufficient if the results do not change.

It is convenient to initiate the trajectories by inserting particles on $P$ with an input current density $\rho_{\text {in }}(\mathbf{x}, t)$. Since a trajectory leaving $P$ has either been initiated there or has intersected $P$ previously, we obtain for the normal component $\rho_{P}(\mathbf{x}, t)$ of the current density on $P$ :

$$
\rho_{P}=\left(\mathcal{L} \rho_{P}\right)+\rho_{\text {in }} .
$$

Here $\mathcal{L}$ is the Frobenius-Perron operator of the TPM, which is defined by

$$
(\mathcal{L} g)(\mathbf{x}, t)=\int_{P} d \mathbf{x}^{\prime} \delta\left(\mathbf{x}-\mathbf{f}\left(\mathbf{x}^{\prime}\right)\right) g\left(\mathbf{x}^{\prime}, t-\tau\left(\mathbf{x}^{\prime}\right)\right) .
$$

We shall consider such $\rho_{\text {in }}$ that equals zero for $t<0$ and either vanishes after a certain positive time, or decays so fast that it can be neglected for long times.

Quite often the motion in one direction-the unstable one-depends at most weakly on the others. Choosing a coordinate system in which $x$ is taken along this direction evolution of $x$ can be well approximated by a one-dimensional map, $x_{n+1}=f\left(x_{n}\right)$. (The price paid is the nonuniqueness of 
$f^{-1}$.) This happens, for example, in strongly dissipative systems and in those analogous to Baker-type maps. In such a situation, Eq. (4) remains valid if $\mathbf{x}$ is replaced by $x$ and $\mathbf{f}$ by $f$, and projecting the densities onto the unstable direction. For simplicity, we restrict our attention to this one-dimensional case.

First we compute the quasistationary distribution. We assume that the system will become quasistationary after some time, i.e., that the distribution decays exponentially but all relative weights remain constant. Normalization of the distribution leads to the time-independent conditionally invariant density [9-11] $\rho_{\mathrm{c}}(x)$. We make the ansatz $\rho_{P}(x, t)$ $=\rho_{\mathrm{c}}(x) e^{-\kappa t}$, where $\kappa$ is the escape rate, and obtain from Eq. (4) the self-consistent equation for the conditionally invariant density

$$
\rho_{\mathrm{c}}(x)=\int_{I} d x^{\prime} \delta\left(x-f\left(x^{\prime}\right)\right) e^{\kappa \tau\left(x^{\prime}\right)} \rho_{\mathrm{c}}\left(x^{\prime}\right)
$$

Here $I$ is the range of the values of $x$. Clearly the solution of this equation for $\kappa$ and $\rho_{\mathrm{c}}$ generically depends on $\tau(x)$, thereby they are different from the corresponding ones of the PM.

For example, let $f$ be the tent map with a possible opening: $f(x)=x / a_{0}$ if $x<a_{0}, f(x)=(1-x) / a_{1}$ if $x>1-a_{1}$, where $a_{0}+a_{1} \leqslant 1$. Furthermore, let $\tau(x)$ be piecewise constant: $\tau(x)=\tau_{0}$ if $x<a_{0}, \tau(x)=\tau_{1}$ if $x>1-a_{1}$. Escape occurs for $a_{0}+a_{1}<1$, when the trajectory leaves the Poincare surface in the interval $x \in\left(a_{0}, 1-a_{1}\right)$. The smooth, nonnegative solution of Eq. (6) is now $\rho_{\mathrm{c}}(x)=1$. By chance $\rho_{\mathrm{c}}(x)$ does not depend on $\tau(x)$, but the equation for $\kappa$ depends on it essentially:

$$
a_{0} e^{\kappa \tau_{0}}+a_{1} e^{\kappa \tau_{1}}=1
$$

and in nonlinear maps $\rho_{\mathrm{c}}(x)$ also depends on it.

For a general treatment we write the formal solution of Eq. (4) as

$$
\rho_{P}(x, t)=\left[(\mathbf{1}-\mathcal{L})^{-1} \rho_{\text {in }}\right](x, t) .
$$

We continue with Laplace transformations in time, since the generalized operator can be written as $(\mathcal{L} g)(t)=\int_{0}^{t} d t^{\prime} L(t$ $\left.-t^{\prime}\right) g\left(t^{\prime}\right)$, with $L(\Delta t) g\left(x, t^{\prime}\right)=\int_{I} d x^{\prime} \delta\left(f\left(x^{\prime}\right)-x\right) \delta\left(\tau\left(x^{\prime}\right)\right.$ $-\Delta t) g\left(x^{\prime}, t^{\prime}\right)$. Its Laplace transform (denoted by $\sim$ ) is $\widetilde{\mathcal{L} g}$ $=\tilde{L} \tilde{g}$ and Eq. (4) yields $\tilde{\rho}_{P}=(1-\widetilde{L})^{-1} \tilde{\rho}_{\text {in }}$. Considering $s$ as a parameter, we can use the eigenfunctions satisfying

$$
\widetilde{L}(s) \varphi_{m}(s) \equiv \int_{I} d x^{\prime} \delta\left(x-f\left(x^{\prime}\right)\right) e^{-s \tau\left(x^{\prime}\right)} \varphi_{m}(s)=\lambda_{m}(s) \varphi_{m}(s)
$$

to expand $\tilde{\rho}_{\text {in }}$ as $\tilde{\rho}_{\text {in }}(s)=\Sigma_{0}^{\infty} a_{m}(s) \varphi_{m}(s)$. Inverse Laplace transformation gives

$$
\rho_{P}(x, t)=\frac{1}{2 \pi i} \int_{c-i \infty}^{c+i \infty} d s \sum_{0}^{\infty} \frac{e^{s t}}{1-\lambda_{m}(s)} a_{m}(s) \varphi_{m}(x, s) .
$$

Each value of $s$ for which $\lambda_{m}(s)=1$ with some $m$ gives a pole in the integrand and a term $e^{s t}$ in $\rho_{P}(t)$. Therefore, the leading asymptotic time dependence is $e^{-\kappa t}$, and hence the escape rate $\kappa$ is determined by the position of the leading pole, i.e.,

$$
\kappa=-s_{0}, s_{0}=\max _{m}\left\{s \text { with } \lambda_{m}(s)=1 \text { and } s \text { real }\right\},
$$

where we assume for simplicity that $s$ is maximal for $m=0$. ( $s$ must be real, otherwise $\rho_{P}(t)$ could not remain positive for all $t$.) Equation (9) together with Eq. (10) corresponds to Eq. (6), however, here we have obtained the result and the decay

$$
\rho_{P}(x, t) \approx\left[1 /-\lambda_{0}^{\prime}(-\kappa)\right] e^{-\kappa t} a_{0} \varphi_{0}(x,-\kappa)
$$

for large times without prescription of $e^{-\kappa t}$.

Now we consider long time averages, under which we mean the following. We take a quantity, which may be a physical observable, that for each trajectory needs a summation of terms $A\left(x_{l}\right)$ taken at every intersection $x_{l}=f^{l}\left(x_{0}\right), l$ $=0,1, \ldots, n-1$ of the trajectory with $P$ within a time duration $t$. Then we average the sum $\sum_{l=0}^{n-1} A\left(x_{l}\right)$ over the trajectories staying in the system until at least time $t$, and finally we take the limit $t \rightarrow \infty$. We consider some examples: If we are interested in the average number of intersections $n$ per time, we set $A(x) \equiv 1$. To get the leading Liapunov exponent describing the exponential deviation of infinitesimally close trajectories we need the logarithm of the derivative of $f^{n}(x)$, i.e., we must set $A(x)=\ln \left|f^{\prime}(x)\right|$.

First we outline the case of the ordinary PM. The average of $\sum_{l=0}^{n-1} A\left(x_{l}\right)$ is obtained by taking into account the contribution of all trajectories present after $n$ iterations. They can be selected by a factor $\int_{I} d x \delta\left(f^{n}\left(x_{0}\right)-x\right)$. After this we divide by the weight of these still present trajectories and arrive at

$$
\begin{aligned}
\left\langle\sum_{l=0}^{n-1} A\left(x_{l}\right)\right\rangle= & \int_{I} d x_{0} \int_{I} d x \delta\left(x_{n}-x\right) \\
& \times \sum_{l=0}^{n-1} A\left(x_{l}\right) \rho_{\text {in }}\left(x_{0}\right) / \int_{I} d x \rho_{P}(x, n) .
\end{aligned}
$$

The denominator is an integral of the density $\rho_{P}(x, n)$ $=\int_{I} d x_{0} \delta\left(x_{n}-x\right) \rho_{\text {in }}\left(x_{0}\right)$ over $x$. Correspondingly, we can write the numerator as an integral of a "weight density of $A^{\prime}, \quad, \quad \sigma_{A}(x, n)=\int_{I} d x_{0} \delta\left(x_{n}-x\right) \sum_{l=0}^{n-1} A\left(x_{l}\right) \rho_{\text {in }}\left(x_{0}\right)$. As is known [19], in the limit of infinite time the time average can be replaced by space average, and $\langle A\rangle_{\infty}$ $\equiv \lim _{n \rightarrow \infty} \frac{1}{n}\left\langle\sum_{l=0}^{n-1} A\left(x_{l}\right)\right\rangle=\int d \mu_{P M}(x) A(x)$. Here the natural measure [13] $\mu_{P M}$ of the PM is defined for a set $H$ as $\mu_{P M}(H)=\lim _{n \rightarrow \infty} \int_{U_{n} \cap H} d x \rho_{P}(x) / \int_{U_{n}} d x \rho_{P}(x)$, and $U_{n}$ $=f^{-n}(I)$ is a series of sets approaching the repeller (the repelling invariant set) for $n \rightarrow \infty$.

The TPM requires two modifications: (i) instead of $n$ we take a time interval of length $t$, (ii) an additional sum over 
the number of intersections $n$ is necessary, since $n$ is generically different for different trajectories. This yields for the weight density of $A$

$$
\begin{aligned}
\sigma_{A}(x, t)= & \sum_{n=0}^{\infty} \int_{I} d x_{0} \int d t_{0} \delta\left(x_{n}-x\right) \\
& \times \delta\left(t-t_{0}-\sum_{j=0}^{n-1} \tau\left(x_{j}\right)\right) \sum_{l=0}^{n-1} A\left(x_{l}\right) \rho_{\text {in }}\left(x_{0}, t_{0}\right),
\end{aligned}
$$

where $\delta\left(t-t_{0}-\sum_{j=0}^{n-1} \tau\left(x_{j}\right)\right)$ demands that the trajectories intersect $n$ times during time $t-t_{0}$. The average value of $\Sigma_{l} A\left(x_{l}\right) / t$ is given asymptotically as

$$
\langle A\rangle_{\infty}=\lim _{t \rightarrow \infty} \frac{1}{t}\left(\int_{I} d x \sigma_{A}(x, t)\right) / \int_{I} d x \rho_{P}(x, t) .
$$

We shall see that this average does not depend on $\rho_{\text {in }}$.

To compute $\sigma_{A}$ we note first that

$$
\sigma_{A}=\mathcal{L}\left(\sigma_{A}+A \rho_{P}\right) .
$$

This is analogous to Eq. (8). Defining $\mathcal{T}=(1-\mathcal{L})^{-1}$ we obtain

$$
\sigma_{A}=\mathcal{T} \mathcal{L} A \mathcal{T}_{\text {in }} .
$$

We introduce $T$ by $(\mathcal{T} g)(t)=\int_{0}^{t} d t^{\prime} T\left(t-t^{\prime}\right) g\left(t^{\prime}\right)$, similarly to the connection of $\mathcal{L}$ and $L$. We can write the Laplace transform of Eq. (16) in terms of the adjoints $\widetilde{L}^{+}$of $\widetilde{L}$ and $\widetilde{T}^{+}$of $\widetilde{T}$. For the backward transformation of this expression, we need the eigenfunctions of $\widetilde{L}^{+}$. The solutions $\psi_{m}(s)$ of $\widetilde{L}^{+}(s) \psi_{m}(s)=\lambda_{m}^{*}(s) \psi_{m}(s)$ are functionals [12-14] (and can be approximated with strongly oscillating functions), due to the fractal nature of the invariant set. We insert an expansion $1=\Sigma_{0}^{\infty} b_{m} \psi_{m}$ and observe that, for large $t$, the most important terms occur when poles induced by $\widetilde{T}^{+*}(s)$ and $\widetilde{T}(s)$ coincide. Thus we obtain for large $t$

$$
\int_{I} d x \sigma_{A}(x, t)=\frac{a_{0} b_{0} t e^{-\kappa t}}{\lambda_{0}^{\prime 2}(-\kappa)} \int_{I} d x \psi_{0}(x,-\kappa) A(x) \varphi_{0}(x,-\kappa) .
$$

[Note that both $\psi_{0}(-\kappa)$ and $\varphi_{0}(-\kappa)$ are real.] The prefactor of Eq. (17) can be expressed by setting $A(x)=\tau(x)$ because for large times $\langle\tau\rangle_{\infty}=1$ according to Eq. (14). Asymptotically we obtain

$$
\langle A\rangle_{\infty}=\frac{1}{\langle\tau\rangle} \int_{I} d x \psi_{0}(x,-\kappa) \varphi_{0}(x,-\kappa) A(x),
$$

where $\langle\tau\rangle \equiv \int_{I} d x \psi_{0}(x,-\kappa) \varphi_{0}(x,-\kappa) \tau(x)$ with the normalization $\int_{I} d x \psi_{0}(x,-\kappa) \varphi_{0}(x,-\kappa)=1$. In analogy with the case of the PM, discussed below Eq. (12), we define the natural measure of the TPM by demanding $\langle A\rangle_{\infty}$ $=\int d \mu(x) A(x)$. Since Eq. (18) is valid for every observable $A$, we see by inspection that the natural measure for infinitesimal intervals is
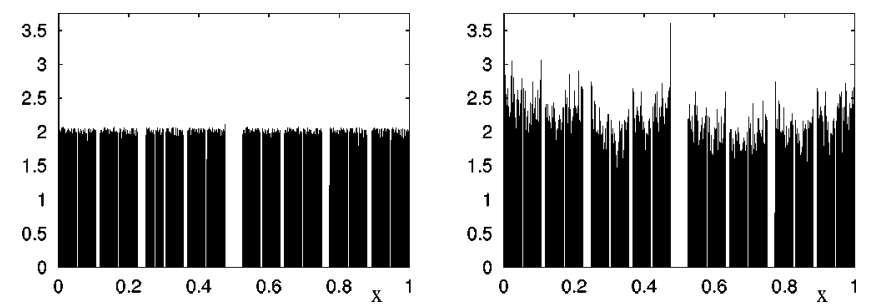

FIG. 1. Fractal distributions $\mu(\Delta x) / \Delta x$ of the natural measures on a finite grid $\Delta x$ when modeling $\mathbf{f}(\mathbf{x})$ by the open tent map $\left(a_{0}\right.$ $=a_{1}=0.475$ ). Left: normal Poincaré map (scale $n$ ). Right: truetime Poincaré map (scale $t$ ), $\tau_{0}=1, \tau_{1}=0.1$.

$$
\mu((x, x+d x))=\frac{1}{\langle\tau\rangle} \psi_{0}(x,-\kappa) \varphi_{0}(x,-\kappa) d x .
$$

A comparison between the natural measure of the PM and the TPM for the tent map (Fig. 1) shows obvious differences. It is clear that the natural measure of the PM and TPM differ significantly, although their dimension $D_{0}$ is the same.

The Liapunov exponent can be written as

$$
\lambda_{\text {Liap }}=\int_{I} d \mu_{P} \ln \left|f^{\prime}(x)\right| .
$$

For $A(x) \equiv 1$ we find $\bar{n}(t)=t\langle 1\rangle_{\infty}=t /\langle\tau\rangle$, thereby

$$
t=\langle\tau\rangle \bar{n}
$$

for large times, which is analogous to Eq. (20).

The leading Liapunov exponent for the repelling tent map is

$\lambda_{\text {Liap }}=\frac{1}{\langle\tau\rangle} \frac{a_{0} \exp \kappa \tau_{0} \ln \left(a_{0}^{-1}\right)+a_{1} \exp \kappa \tau_{1} \ln \left(a_{1}^{-1}\right)}{a_{0} \exp \kappa \tau_{0}+a_{1} \exp \kappa \tau_{1}}$.

In this example we see the irrelevance of $\tau$ for $\kappa=0$ (no transient chaos), so that $\langle\tau\rangle$ only sets the time scale. On the other hand, for $\kappa>0 \lambda_{\text {Liap }}$ is not invariant to changes of $\tau_{0}$ relative to $\tau_{1}$, proving again that $\tau$ is a relevant quantity in transient chaos.

The comparison of the behavior of the flow and the map shows that the occurrence of criticality can change when turning from the map to the real system. A state of a system is called critical if the natural measure is concentrated on a subset of the repeller, while the invariant measure is distributed on the whole repeller. In such situations there are two conditionally invariant measures with different escape rates $[15,16,19]$. Here we use the piecewise parabolic 1D map [15] that is defined on the interval $[0,1]$ by its inverse branches $f_{l}^{-1}(x)=(x+d \cdot x(1-x)) / 2 R \quad$ (lower branch), $f_{u}^{-1}(x)=1$ $-f_{l}^{-1}(x)$ (upper branch) and choose $\tau(x)=1+\tau_{x} \cdot(x-1 / 2)$. When increasing $\tau_{x}$ at a certain value the escape rates change order. This is evidently a breakpoint in Fig. 2, which shows the leading (smaller) one of the escape rates. Above that point criticality disappears. This will be explored in more detail elsewhere.

If $P$ and the dynamics on it are periodic $P$ can be reduced to a unit cell with periodic boundary conditions. In this case 


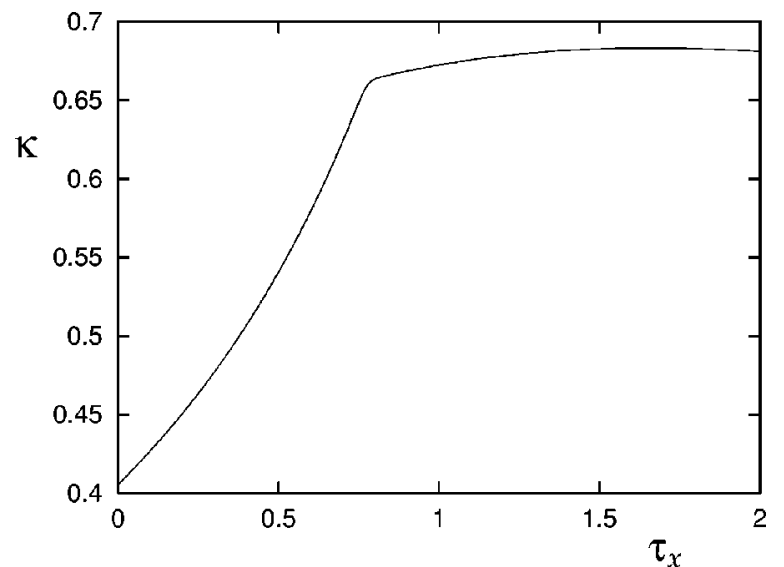

FIG. 2. The leading escape rate of the piecewise parabolic map as a function of the derivative of $\tau(x), \tau_{x}$.

both the PM and TPM can be separated into a reduced map (which maps the unit cell into itself), plus a shift $\Delta(x)$ describing the transit between the cells $[17,18]$. Such systems can be characterized by the drift speed and diffusion coefficient. If particles can be lost from the point of view of diffusion by absorption, chemical reaction or escape in directions transverse to the extension of the system, we refer to transient diffusion [19-21]. We set $A=\Delta$ and we obtain a shift density $\sigma_{\Delta}$ in analogy to the procedure above: $\sigma_{\Delta}$ $=\mathcal{T} \mathcal{L} \Delta \mathcal{T}_{\rho_{\text {in }}}$. We then determine the drift speed as the normalized shift per time

$$
v=\lim _{t \rightarrow \infty} \frac{\text { average of }\{S(t)\}}{t}=\frac{1}{\langle\tau\rangle}\langle\Delta\rangle_{\infty} .
$$

As an example of the essential role of averages over the natural measure, we consider a diffusive system on a onedimensional lattice and assume that the reduced map is the tent map. We assume furthermore a microscopic process de- termining whether and by how much a particle jumps to the left or to the right on the lattice. We consider a lattice with period 1 and a change of the coordinate by \pm 1 depending on the location in the subinterval $[0,1]$, namely $\Delta(x)=-1$ if $x \in\left[0, a_{0}\right], \Delta(x)=1$ if $x \in\left[a_{1}, 1\right]$. To calculate the average speed or the diffusion coefficient an average over long trajectories is required. The average speed is

$$
v=\frac{1}{\langle\tau\rangle} \frac{a_{1} \exp \kappa \tau_{1}-a_{0} \exp \kappa \tau_{0}}{a_{1} \exp \kappa \tau_{1}+a_{0} \exp \kappa \tau_{0}} .
$$

Again if $\kappa=0$ (nontransient chaos) the return time $\tau$ sets the time scale only, and the PM and TPM give the same result. But for $\kappa>0$ (transient chaos) even the sign of the speed can change when computing it with the usual PM, i.e., when setting $\tau(x) \equiv\langle\tau\rangle \equiv$ const. Results for the diffusion coefficient will be published elsewhere.

In conclusion, we have shown that the return time $\tau$, i.e., the time between two successive intersections on the Poincaré surface $P$, is a relevant quantity in transient chaos. The usual Poincaré map does not reflect the long-time averages of the flow satisfactorily and can even be completely misleading. The solution is to use a true-time Poincare map TPM and its generalized Frobenius-Perron operator (5), where we can also define conditionally invariant measure and natural measure. Escape rate, Liapunov exponents, drift speed, etc., depend significantly on $\tau(x)$ and are described correctly only by using the TPM. Therefore the necessary generalization of the normal Poincare map is the true-time Poincare map if the system in question is a repeller.

We thank J. Bene, R.O. Jones, and G. Eilenberger for helpful discussions and comments on the manuscript. This work has been supported in part by the Hungarian National Scientific Research Foundation under Grant No. OTKA T017493. Z.K. thanks G. Eilenberger for the hospitality of the IFF, Forschungzentrum Jülich, where part of this work was done.
[1] P. Gaspard, and G. Nicolis, Phys. Rev. Lett. 65, 1693 (1990).

[2] T. Gilbert, C.D. Ferguson, and J.R. Dorfman, Phys. Rev. E 59, 364 (1999).

[3] T. Tél and J. Vollmer, in Encyclopedia of Mathematical Sciences, edited by D. Szász (Springer-Verlag, Berlin, 2000), Vol. 101, pp. 367-418.

[4] G. Károlyi and T. Tél, Phys. Rep. 290, 125 (1997).

[5] Z. Neufeld, C. López, E. Hernández-García, and T. Tél, Phys. Rev. E 61, 3857 (2000).

[6] J.-P. Eckmann and D. Ruelle, Rev. Mod. Phys. 57, 617 (1985).

[7] P. Gaspard, Phys. Rev. E 53, 4379 (1996).

[8] L.A. Bunimovich, Zh. Éksp. Teor. Fiz. 89, 1452 (1985) [Sov. Phys. JETP 62, 842 (1985)].

[9] G. Pianigiani and J.A. Yorke, Trans. Am. Math. Soc. 252, 351 (1979)
[10] G. Pianigiani, J. Math. Anal. Appl. 82, 75 (1981).

[11] T. Tél, in Directions in Chaos, edited by Hao Bai-Lin (World Scientific, Singapore, 1990), Vol. 3, pp. 149-211.

[12] H.H. Rugh, Ph.D. thesis, Niels Bohr Institute, 1992.

[13] H. Kantz and P. Grassberger, Physica D 17, 75 (1985).

[14] P. Gaspard, J. Phys. A 25, L483 (1992).

[15] A. Németh and P. Szépfalusy, Phys. Rev. E 52, 1544 (1995).

[16] H. Lustfeld and P. Szépfalusy, Phys. Rev. E 53, 5882 (1996).

[17] S. Grossmann and H. Fujisaka, Phys. Rev. A 26, 1779 (1982).

[18] T. Geisel and J. Nierwetberg, Z. Phys. B: Condens. Matter 56, 59 (1984).

[19] Z. Kaufmann, H. Lustfeld, A. Németh, and P. Szépfalusy, Phys. Rev. Lett. 78, 4031 (1997).

[20] P. Collet and S. Martinez, Nonlinearity 12, 445 (1999).

[21] I. Claus and P. Gaspard, Phys. Rev. E 63, 036227 (2001). 